\title{
ALLOPLASTIC PUTTY BONE GRAFT- A SHORT REVIEW
}

\author{
Dr. Nisha Mary Jose, Dr. Ashok Kumar. S, Dr. Jaideep Mahendra, Dr. Abirami Nayaki Rao.P \\ Department of Periodontology, Faculty of Dentistry
}

Meenakshi Ammal Dental College and Hospital, Meenakshi Academy of Higher Education and Research, Alapakkam Main Road, Chennai, Tamilnadu, India

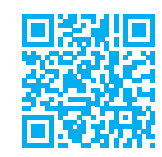

DOI:10.37841/jidam_2021_V8_I1_08

Address for Correspondence:

Dr. S. Ashok Kumar, MDS.,

Associate Professor, Department of Periodontology,

Faculty of Dentistry, Meenakshi Ammal Dental College and Hospital,

Meenakshi Academy of Higher Education and Research,

Alapakkam Main Road, Chennai, Tamilnadu, India

Emailid: drashok.perio@madch.edu.in

Received: 16.02.2021 First Published: 15.03.2021

Accepted: 14.03.2021

Published: 27.03.2021

\section{ABSTRACT}

The shift in therapeutic concepts from resection to regeneration has influenced the advancement in grafting materials. The enormous progress in the field of biomaterial science, the risk of infectious diseases transmission and finally, efforts to reduce morbidity and cost has led research into the development of a variety of synthetic graft materials as alternatives. Synthetic bone grafts have been shown to produce greater clinical bone defect fill as a bone graft substitute.

KEYWORDS: Alloplastic Bone graft, Guided bone regeneration 
Nisha et al: Alloplastic Putty Bone Graft

\section{INTRODUCTION:}

Healing of bone is a multifactorial and complex process. With the current revolution in biotechnology and tissue engineering, the number of options for bone grafting have continued to grow. Factors such as type of graft, site of preparation, mechanical strength, vascularity and pore size of the material are involved in the successful incorporation of a grafted material. New developments in material science offers a range of synthetic replacements for natural grafts. ${ }^{1}$

\section{BONE GRAFT CHARACTERISTICS:}

- Osteoinduction - ability of the graft to recruit and induce mesenchymal stem cells to differentiate into mature bone-forming cells.

- Osteoconduction - cellular invasion of the graft.

- Osteointegration - functional integration of the graft with the host tissue through new bone formation.

- Osteogenesis - recruitment of osteoblast precursors and growth factors at sites of augmentation. $^{2}$

Synthetic grafting materials or alloplastic materials are shown to possess characteristics such as osteoconduction and osteointegration. The ideal synthetic graft material should be biocompatible, support new bone growth and undergo remodelling. Modulus of elasticity, toughness, and compressive strength compared with host cortical or cancellous bone are the other features of a bone graft. The most common alloplastic materials are tricalcium phosphates(TCP), bioactive glass, hydroxyapatite(HA) and dicalcium phosphates.

\section{PUTTY BONE GRAFT:}

Various studies have reported the superior manageability, hemostatic and osteoconductive properties of bioactive glass and may act to retard epithelial downgrowth. NovaBone ${ }^{\circledR}$ Dental Putty in Syringe* is a new, next generation calcium-phosphosilicate (CPS) bone graft material based on NB's bioengineered technology platform that offers enhanced handling characteristics with unparalleled performance. CPS bone graft material is built from a bioactive glass with additives like polyethylene glycol (PEG) and glycerin to improve handling and efficacy. The particulate and binder are provided premixed as a pliable cohesive material. On implantation, the binder is absorbed to permit tissue infiltration between the bioglass particles. The particles are slowly absorbed and replaced by new bone tissue during the healing process. This osteostimulation results in new bone formation throughout the grafted site at rates faster than those seen with other synthetics.

\section{$*_{\text {NovaBone }}{ }^{\circledR}$ Dental Putty in Syringe (NovaBone} Products, LLC)

Built on a CPS platform, NB putty demonstrates superior performance characteristics that are a result of multiple physical and chemical interactions: Osteostimulation is an active process, and CPS dental putty acts as a bone matrix and encourages differentiation of new bone cells at the site.

Putty has been approved by Food and Drug Administration and CE dental indications in 2007. It was the first synthetic putty that required no handling or manipulation and the first to be available in a significantly simplified cartridge delivery system. Calcium phosphosilicate putty minimizes graft wastage and reduces chair-side time. Unlike other synthetic grafts that are bioinert, CPS putty belongs to the class of bioactive regenerative materials that not only act as an osteoconductive scaffold but also interacts with the surrounding tissues and imparts an osteostimulatory effect. The viability and proliferation potential of osteoblasts has been shown to be exemplified in the presence of CPS particles. These CPS particles contain an increased osteocalcin and alkaline phosphatase levels providing a favourable site for bone formation ${ }^{3}$ (Figure 1 and 2).

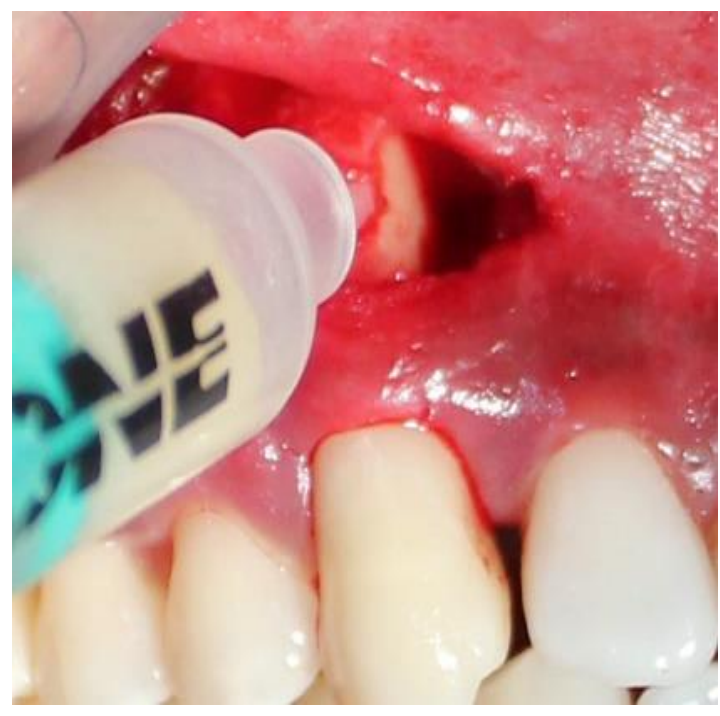

Figure 1: Novabone putty bone graft in syringe 
Nisha et al: Alloplastic Putty Bone Graft

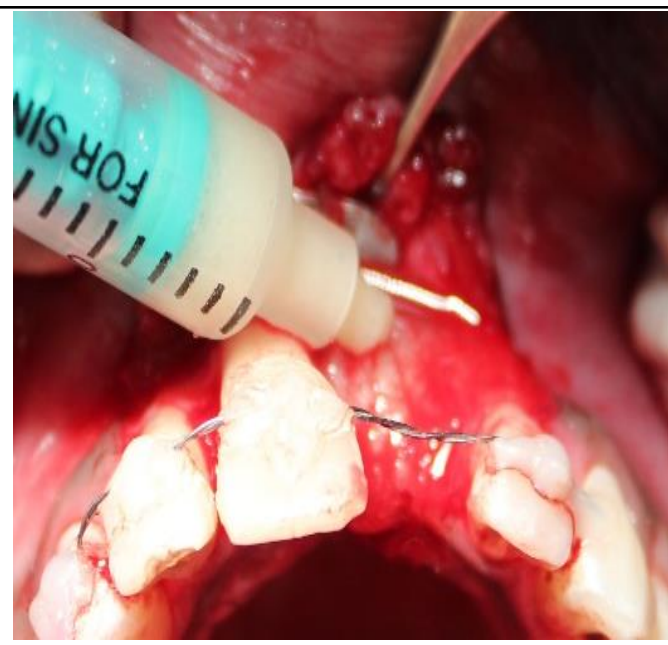

Figure 2: Easy adaptation of bone graft into the defect site

\section{COMPOSITION:}

The CPS putty is composed of a bimodal particle distribution of CPS (active ingredient), with PEG as an additive and glycerine as the binder. The volume of the active ingredient is approximately $70 \%$. The components are premixed, and the putty is delivered in a ready-to-use state. Both PEG and glycerin are water soluble and are engineered to be absorbed from the site in 3 to 5 days. The putty is tan in colour after sterilization. ${ }^{3}$

\section{ADVANTAGES OF THE CPS PUTTY:}

- Ideal for minimally invasive surgeries, hard to access defects, immediate implant surgeries, osteotome sinus surgeries, etc.

- Unique to CPS putty, osteostimulation increases the rate of bone formation.

- Putty will not washout from the graft site during irrigation and suction.

- During manipulation CPS putty does not adhere to surgical gloves or instruments.

- CPS putty has no risk of antigenic response or disease transmission.

- CPS putty appears radiodense on the radiograph.

- It is stable at room temperatures $\left(25^{\circ} \mathrm{C}\right)$ and does not require any refrigeration. ${ }^{4}$

\section{APPLICATION IN PERIODONTICS:}

- Immediate implant surgeries
- $\quad$ Ridge augmentation

- Sinus elevation

- Cystic defect

- Fenestration and dehiscence defect ${ }^{5}$

\section{FATE OF CPS PUTTY GRAFT:}

Upon implantation, clinically the binder gets absorbed within 24 to 72 hours creating a three-dimensional porous scaffold that facilitates active movement of blood and tissue fluids through the matrix. There is an initial burst of calcium and phosphate ions, which is provided by the interaction of small CPS particles with blood. Numerous calcium phosphate nodules are formed due to this interaction that mature individually to form bone throughout the defect. Subsequently, the larger particles react and continue the process of bone regeneration. Bone density analysis suggests that there is a steady increase in bone density for 6 months. ${ }^{3}$

\section{CONCLUSION:}

Modalities of treatment that involve less invasive, more reproducible and less technique sensitive procedures and biomaterials are needed. New developments towards a more individualized and personalized bone regeneration therapy are being evolved.

\section{FINANCIAL SUPPORT AND SPONSORSHIP}

Nil

\section{CONFLICTS OF INTEREST}

There are no conflicts of interest.

\section{REFERENCES:}

1. Eppley BL, Pietrzak WS, Blanton MW. Allograft and alloplastic bone substitutes: a review of science and technology for the craniomaxillofacial surgeon. J Craniomaxillofac Surg 2005;16(6):981-989.

2. Sheikh Z, Sima C, Glogauer M. Bone replacement materials and techniques used for achieving vertical alveolar bone augmentation. Materials 2015;8(6):2953-2993.

3. Doddarayapete N Umashankar, Kranthi S Kumar, R Mahesh Kumar, Narasimhamurthy Srinath, Chaithra Patil. Efficacy of Calcium Phosphosilicate as Graft Material in Bony Defects. Int J Oral Care Res. 2018;6(1):17-23. 
Nisha et al: Alloplastic Putty Bone Graft

4. Mahesh L, Salama M, Kurtzman GM. Extraction of an impacted maxillary canine with immediate implant placement. Inside dentistry 2012;8(3):24.

5. Kotsakis G, Chrepa V, Katta S. Practical application of the newly introduced natural bone regeneration (NBR) concept utilizing alloplastic putty. Int $\mathbf{J}$ Oral Implantol Clin Res 2011;2(3):145-149. 